\title{
Narrativas visuales desde la hibridación de los espacios habitados por agentes diversos de la Facultad de Educación (UPV-EHU)
}

\section{Visual narratives from the hybridization of the spaces inhabited by different agents of the Faculty of Education (UPV-EHU)}

\section{Maitane Picaza Gorrotxategi}

Universidad del País Vasco (UPV/EHU)

maitane.picaza@ehu.eus

\section{Amaia Eiguren Munitis}

Universidad del País Vasco (UPV/EHU)

amaia.eiguren@ehu.eus

\section{María Dosil Santamaría}

Universidad del País Vasco (UPV/EHU)

maria.dosil@ehu.eus

\author{
Miriam Peña Zabala \\ Universidad del País Vasco (UPV/EHU) \\ miriam.pena@ehu.eus \\ Israel Alonso Sáez \\ Universidad del País Vasco (UPV/EHU) \\ israel.alonso@ehu.eus
}

Recibido: 15/09/2020 Revisado: 18/10/2020

Aceptado: 09/12/2020 Publicado: 14/12/2020

\section{Resumen:}

El espacio nunca es neutro ya que es habitado por sujetos que van forjando identidades en los lugares que ocupan. Dentro de este contexto la Facultad de Educación de Bilbao (UPV/EHU) ha llevado a cabo en el curso 2019-2020 un proyecto enfocado a la reflexión conjunta de diferentes agentes educativos sobre los espacios. El proyecto tiene como

\section{Sugerencias para citar este artículo:}

Picaza Gorrotxategi, Maitane; Eiguren Munitis, Amaia; Dosil Santamaria, María; Peña Zabala, Miriam y Alonso Sáez, Israel (2020). Narrativas visuales desde la hibridación de los espacios habitados por agentes diversos de la Facultad de Educación (UPV-EHU). Tercio Creciente (Monográfico extraordinario IV), (pp. 59-70), https://dx.doi.org/10.17561/rtc.extra4.5749

PICAZA GORROTXATEGI, MAITANE; EIGUREN MUNITIS, AMAIA; DOSIL SANTAMARIA, MARÍA; PEÑA ZABALA, MIRIAM y ALONSO SÁEZ, ISRAEL. Narrativas visuales desde la hibridación de los espacios habitados por agentes diversos de la Facultad de Educación (UPV-EHU). Tercio Creciente (Monográfico extraordinario IV), diciembre 2020, pp. 59-70, https://dx.doi.org/10.17561/rtc.extra4.5749 
objetivo ahondar en las inquietudes personales de las personas participantes recogiendo sus voces a partir de dos preguntas generadoras: ¿Cuál es la razón por la que estamos en el proyecto?, ¿Qué nos ha llevado a tomar parte en este proyecto? El hecho de recoger las voces ayuda a crear un diálogo horizontal entre la palabra y la imagen, posicionando las mismas en un mismo nivel como vehículo narrador de las historias que transitan desde una visión personal a una visión más colectiva y sitúa a las personas en continua relación con los espacios.

\section{Abstract}

Space is never neutral as it is inhabited by subjects who forge identities in the places they occupy. Within this context, the Faculty of Education of Bilbao (UPV/EHU) has carried out a project in the academic year 2019-2020 focused on the joint reflection of different educational agents on the spaces. The project aims to deepen in the personal concerns of the participants by collecting their voices from two generating questions: What is the reason why we are in the project, what has led us to take part in this project? The fact of collecting the voices helps to create a horizontal dialogue between the word and the image, positioning them on the same level as a narrative vehicle of the stories that move from a personal vision to a more collective vision and places people in continuous relationship with spaces.

\section{Palabras clave}

Espacio, reflexión, identidad, fotoensayo, imagen.

\section{Keywords}

Space, reflection, identity, photo essay

\section{Sugerencias para citar este artículo:}

Picaza Gorrotxategi, Maitane; Eiguren Munitis, Amaia; Dosil Santamaria, María; Peña Zabala, Miriam y Alonso Sáez, Israel (2020). Narrativas visuales desde la hibridación de los espacios habitados por agentes diversos de la Facultad de Educación (UPV-EHU). Tercio Creciente (Monográfico extraordinario IV), (pp. 5970), https://dx.doi.org/10.17561/rtc.extra4.5749

PICAZA GORROTXATEGI, MAITANE; EIGUREN MUNITIS, AMAIA; DOSIL SANTAMARIA, MARÍA; PEÑA ZABALA, MIRIAM y ALONSO SÁEZ, ISRAEL. Narrativas visuales desde la hibridación de los espacios habitados por agentes diversos de la Facultad de Educación (UPV-EHU). Tercio Creciente (Monográfico extraordinario IV), diciembre 2020, pp. 59-70, https://dx.doi.org/10.17561/rtc.extra4.5749 


\section{Introducción}

Este trabajo es parte una investigación más amplia que se presenta junto con otras dos partes, y se compone como un puzzle de tres piezas inspiradas en audios, cartografías y voces de un grupo de profesorado universitario, alumnado y personal administrativo y de servicios de la Universidad del País Vasco (UPV/EHU), que reflexiona cómo resignificar el espacio social de la propia Facultad de Educación de Bilbao (UPV/EHU). Las tres partes del proyecto toman como referencia el concepto de hibridación, el cual puede devenir en la mejora de las relaciones y en la conexión significativa de la comunidad educativa que habita y transita por la Universidad (Lie, 2009).

Este proyecto es concedido dentro del marco de la sostenibilidad y promovido por la convocatoria 2019/2020 de Campus Bizia Lab, en el cual se fomentan los procesos colaborativos entre personal de administración y servicios, estudiantes y profesorado, con objeto de responder a retos de sostenibilidad dentro de la propia Universidad.

Dentro de este marco, entendemos que nos comportamos y relacionamos conforme a los espacios que transitamos y que a su vez son condicionante de nuestras acciones y ausencias (Gupta y Ferguson, 2008). Por lo tanto, el espacio social transitado se convierte en un tercer maestro/a sutil que nos conforma y transforma en el contexto cotidiano y por ende en el universitario (Gutierrez, 2005).

Con este marco de fondo, el objetivo del proyecto se centra en reflexionar sobre el uso del espacio social que conforma la Facultad de Educación de Bilbao (UPV/EHU) y las relaciones que en ella se articulan. Asimismo, la comunidad educativa lejos de ser un ente estático y homogéneo se convierte en un espacio plástico y permeable de acción donde diferentes actores educativos y sociales forjan su identidad (Echevarría, 2003). Nuestros cuerpos van colonizando los espacios educativos (Fassina y Torres, 2013). En este sentido, creemos que la comunidad educativa puede determinar un sentimiento de pertenencia que se expresa en un reconocimiento inconsciente de la existencia del "nosotras". En este contexto ocurre un cruce de caminos entre espacios híbridos estáticos y móviles.

Desde este prisma poliédrico se quiere poner el acento en las personas participantes del proyecto para generar una red de aprendizaje alternativo al tradicional (Olmos, Sánchez y Correa, 2016). Concretamente, el grupo está formado por 
trabajadores administrativos, alumnado y profesorado de expresión plástica, expresión musical, educación social y pedagogía. Se quiere ahondar en las inquietudes personales de las personas participantes recogiendo sus voces. De esta forma, se crea un diálogo horizontal entre la palabra y la imagen posicionadas en un mismo nivel como vehículo narrador de las historias generadas por las personas en continua relación con los espacios sociales y educativos.

\section{Metodología}

Teniendo como punto de partida la omnipresencia de la imagen en la sociedad actual (Yañez-Urbina, Figueroa, Soto y Sciolla, 2018), el proceso de construcción se basa en la narrativa visual como vehículo para fomentar la reflexión crítica de las realidades que nos rodean, contemplando nuestro entorno desde diferentes prismas (Córdoba, 2007; Mannay, 2007).

En este proceso participaron 18 personas, 13 docentes, 2 personas de personal de administración y servicios y 3 alumnos/as, con edades comprendidas entre los 19 y los 60 años, los cuales conformaron el equipo motor multidisciplinar del proyecto. Además, se pudieron contrastar diferentes realidades territoriales puesto que 14 personas estaban vinculadas a la Facultad de Educación de Bilbao (Vizcaya) y 4 personas a la Facultad de Educación, Filosofía y Antropología de San Sebastián (Guipúzcoa).

Con el objetivo de ahondar en las inquietudes personales de las personas participantes se plantearon las siguientes preguntas: ¿Cuál es la razón por la que estamos en el proyecto?, ¿Que nos ha llevado a tomar parte en este proyecto?. De esta forma, se creó un diálogo horizontal entre la palabra y la imagen posicionadas en un mismo nivel como vehículo narrador de las historias generadas por las personas en continua relación con los espacios. Asimismo, se pudo transitar desde la visión individual y personal hacia la visión colectiva de grupo.

\section{Construcción y desarrollo de la visión colectiva}

Para poder transitar desde la visión individual a la colectiva el proceso de desarrollo fue forjándose de forma escalonada en diferentes fases: 


\section{Fase 0-Relatos Personales}

Las personas participantes comenzaron su reflexión realizando una introspección personal sobre los motivos y expectativas puestas en la participación del proyecto.

Entre ellas destacan en primer lugar los intereses propios del equipo de trabajo ligados a la implicación personal con el equipo motor "continúo en este proyecto por el aspecto relacional y emocional que me vincula a las profesoras" (R_P2). Además, tanto la temática del proyecto "el tema de investigación me parece muy interesante y necesario" (R_P3), su idiosincrasia unida con la construcción de las identidades "el espacio, sin quererlo, nos configura y nos hace olvidar que todos y todas nosotras también formamos parte de sus dinámicas, y que podemos contribuir a su propia reorganización" (R_P9) como la oportunidad de crear espacios de reflexión "este proyecto me invitaba a mirar más allá de lo naturalizado y a interpretar que los espacios guardan unas lógicas de poder que contribuyen a construir nuestras propias identidades"(R_P9) se convierten en ejes motivadores de participación.

Asimismo, la motivación ligada a la implicación profesional "soy gestora de los espacios de la facultad por lo que creo es necesario implicarse en el proyecto" (R_P10), la labor docente "siento que el espacio condiciona mi labor y la encamina a una determinada docencia sin ser yo consciente de ello" (R_P1) e investigadora "estamos trabajando la influencia del espacio a través del grupo de investigación" (R_P5) aparece latente.

En esta misma línea cabe resaltar el carácter innovador del proyecto desde su visión holística. La participación de diferentes perfiles (docentes, personal administrativo, alumnado) es un punto de anclaje en el cual las personas participantes encuentren un espacio compartido de reflexión conjunta "es una oportunidad de colaborar con otros compañeros/as en un proyecto de investigación, practicar la escucha atenta, abrirnos a nuevas experiencias en otros contextos...se me hacía muy atractivo la perspectiva inclusiva de juntarnos personal de la administración y servicios, alumnado y profesorado" (R_P3) tomando conciencia de la importancia de los espacios habitados y la relación que se crea con ellos "el reflexionar y dar a conocer las relaciones que mantenemos con los espacios educacionales (y más en concreto en una facultad de educación) nos hablan de cómo habitamos esos espacios y nos ayuda a entender la relación que nuestro cuerpo tienen con el saber" (R_P7). 


\section{Fase 1: La imagen como representación de la realidad}

En la primera fase se tomó una fotografía que evoca una escena descontextualizada inspirada en la obra de Eshter, Ferrer. Desde las limitaciones en tiempos de COVID-19 se buscó una analogía para explicar la percepción que el espacio educativo de la Facultad de Educación de Bilbao provoca en las personas participantes. Siempre desde la experiencia personal y la realidad vivida.

En la fotografía un baño blanco, frío y aséptico es el escenario elegido. La facultad de Educación de Bilbao se sitúa en un edificio relativamente nuevo (2011) pero que según las personas participantes no transmite su larga historia (antes estuvo situado en otros puntos de la villa Bilbaína como Escuela de Magisterio). Tampoco transmite la esencia de un edificio donde las personas trabajan por la transformación social desde una base humanística. Como recurso con grandes posibilidades no está aprovechado.

Al fondo, una gran ventana abierta deja ver una pared que a su vez enclaustra ventanas con rejas. La ambivalencia de la metáfora representa, por un lado, la idea anterior donde la ventana lejos de airear termina en barrotes. La Facultad de Educación es un edificio donde los barrotes físicos y simbólicos impiden la sensación de apertura y de ventilación. Por otro lado, y desde la perspectiva inversa, la ventana como símbolo de libertad se abre a la oportunidad de oxígeno y de nuevas ideas o proyectos. Un patio común donde poder dialogar desde dentro hacia afuera.

En el centro, una mujer que puede recordar a El Pensador de Rodin (1881) carga de contenido la escena. Por una parte, se trata de un cuerpo que rompe con la heteronormatividad establecida, un cuerpo de mujer adulta que aporta la nota de color. Por otra parte, alude a todas las maestras que a lo largo de la historia han contribuido en el sistema educativo. Mujeres trabajadoras, cultas y sabias. Mujeres que han trabajado en su triple rol de maestras, cuidadoras y ciudadanas. La mujer de la foto viste zapatillas y gafas como un reflejo de pertenecer al espacio dicotómico privado-público. Además, la figura reposa sobre una columna de libros que contrastan con un moderno ordenador que hace de tránsito hacia una era digitalizada cada vez más feroz. Al igual que ocurre con la ventana como metáfora, en parte, depende de nosotras y de los agentes educativos hacer un uso constructivo o no de las nuevas tecnologías con sus limitaciones y posibilidades. 


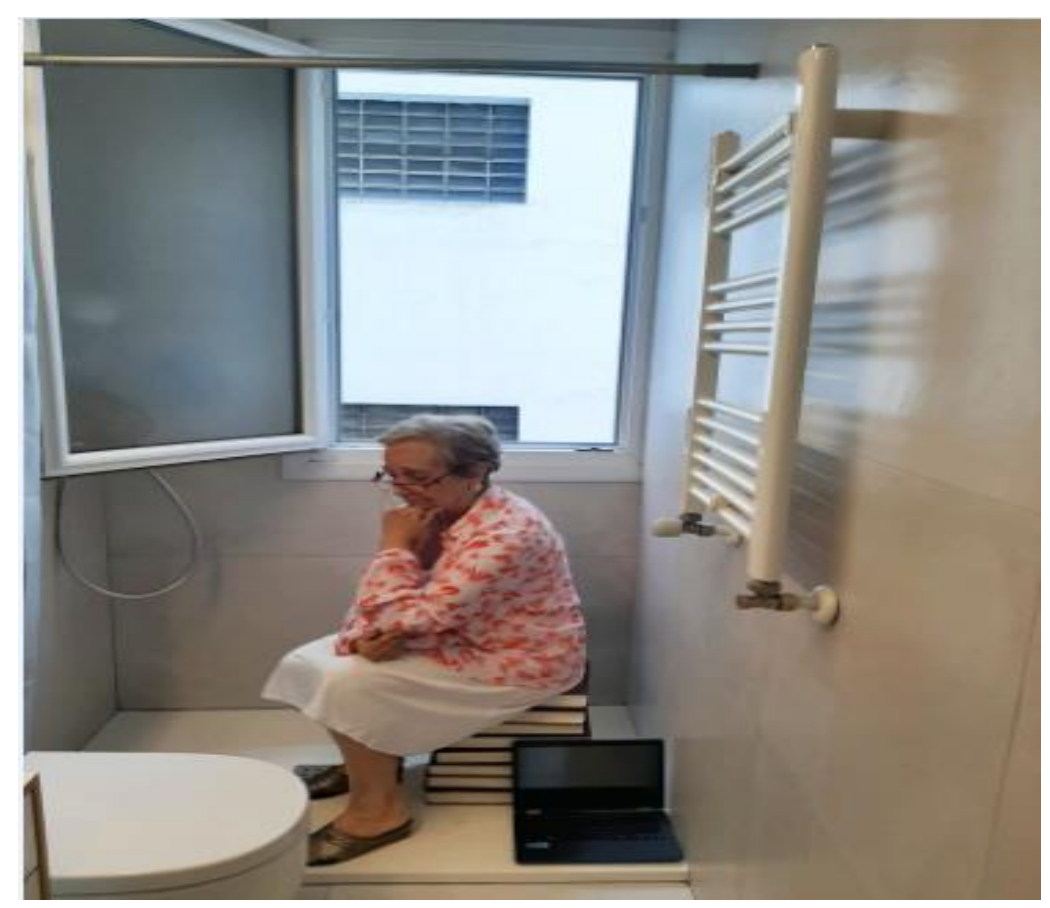

Imagen1. Fotografía completa. Fuente: Elaboración propia.

\section{Fase 2: Visión Individual}

En cuanto a la fotografía (imagen 1) se les pidió a los/las participantes que eligieran un fragmento de la foto que les atrajera uniéndose (imagen 2) con la reflexión de los espacios como medios de conexión entre personas.

En algunos casos los fragmentos fueron acompañados de ideas que profundizan en la reflexión. Dentro de ellas destaca el ser y no ser, la dicotomía entre parecer y ser unido con la necesidad de libertad:

"la utilidad del espacio", "definición del espacio", "la vida del espacio y su relación", "la huella que dejamos en el espacio", "en la fotografía salen formas muy similares pero son diferentes dependiendo si se observa desde el espacio interior o exterior", " para mí la ventana muestra salida, oportunidades, no sabes lo que es exactamente pero quieres saber más", "la propia sombra, la proyección de cada uno/a", "proyección, espacios y vida, todos tenemos un proyecto", "una mirada a los espacios y a la vida, que te ayuda a reflexionar", "la ventana da a un 
patio, el patio es un espacio común interior. La ventana tiene una vista limitada, pero se ve otra ventana enfrente, abramos ventanas"

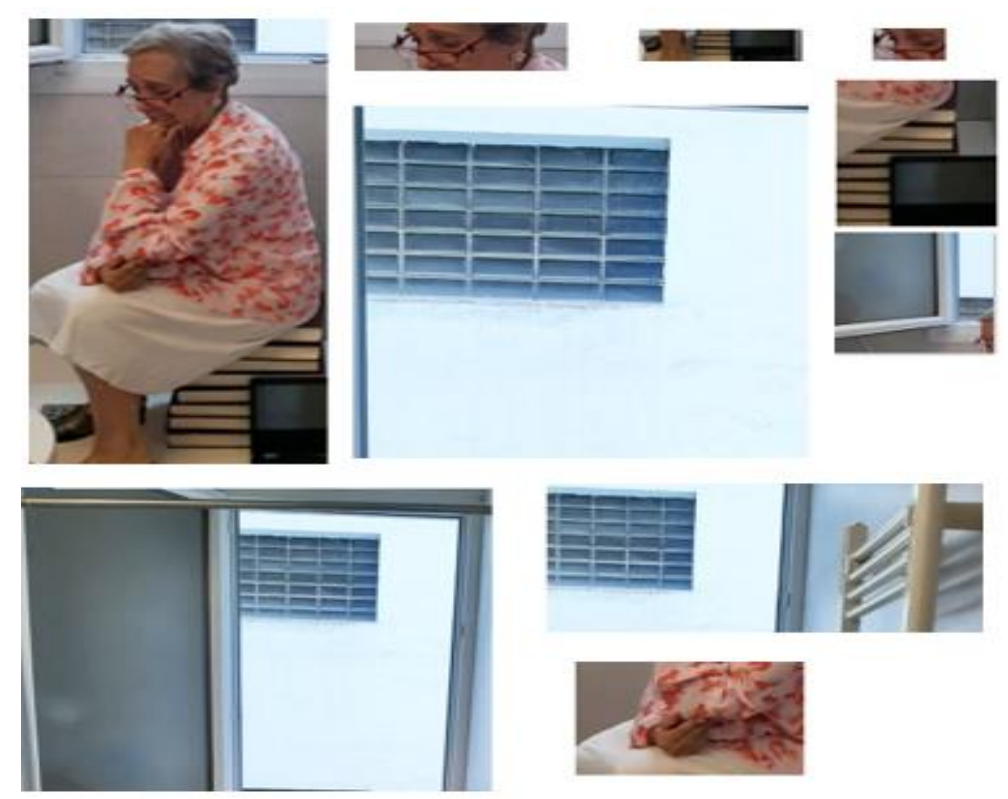

Imagen 2: unión de fotografías. Fuente: Elaboración propia.

\section{Fase 3: Visión Compartida}

En cuanto a la fase tres, el collage (imagen 3), cabe destacar que se construye desde la visión individual que pasa a ser colectiva. De algún modo volvemos a los relatos del principio, pero esta vez el relato se ve modificado por la visión narrativa de un grupo (los y las participantes de este proyecto) que deriva en una nueva visión colectiva. 


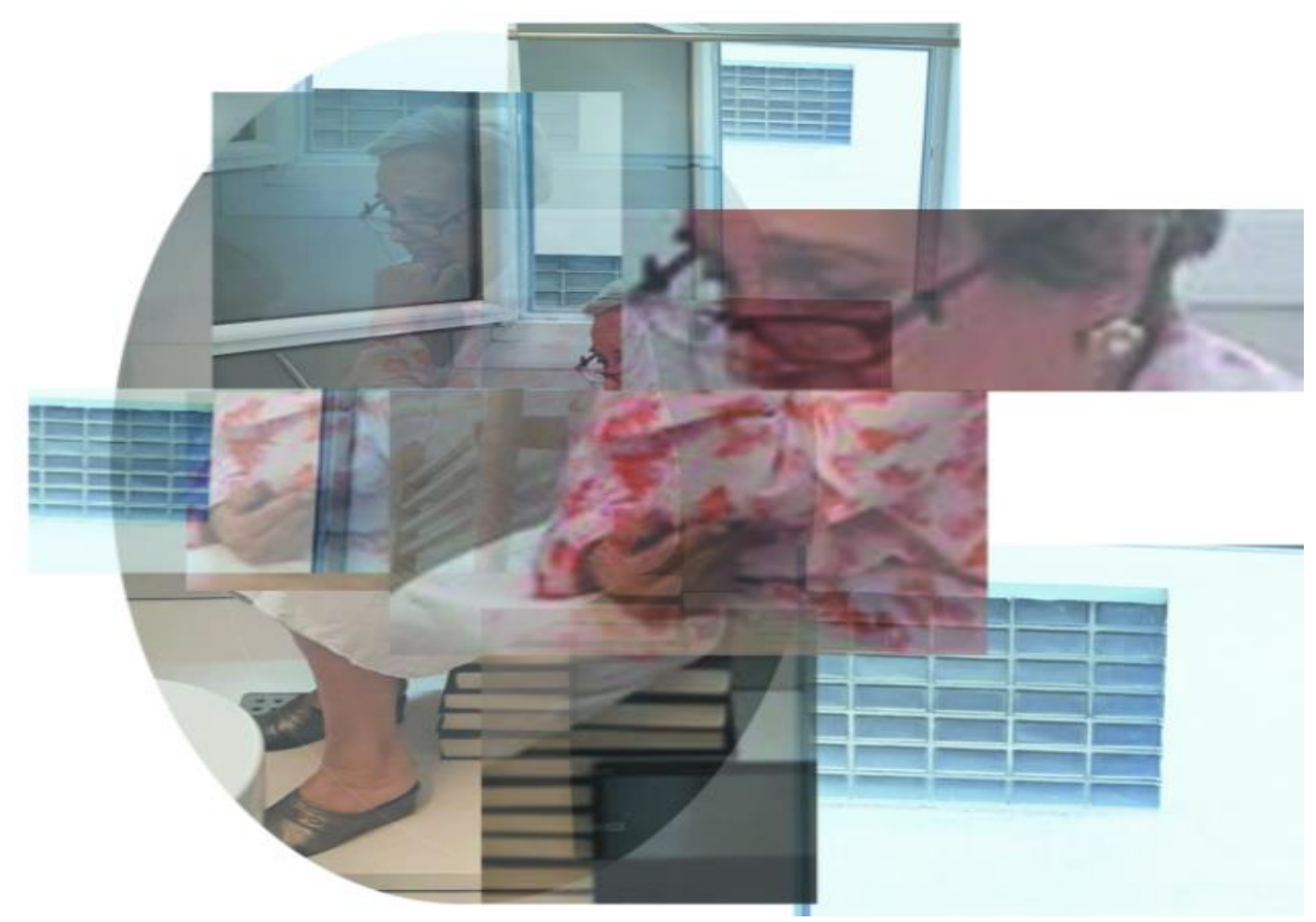

Imagen 3. Collage de los fragmentos elegidos por las personas participantes Fuente: elaboración propia

\section{Conclusión}

La narrativa visual comienza en el interior de la persona desde la experiencia vivida. A través del diálogo, el proceso irónicamente inacabado, culmina en un collage que aglomera y engloba el conjunto de las visiones para derivar en una narración visual de interpretación colectiva.

Fragmentos de una pensadora, ventanas, barrotes, libros y ordenador se interponen en una imagen final llena de contrapuntos. Con este mosaico final volvemos al punto de inicio, pero con una visión compartida y renovada donde la reflexión individual da paso a la construcción de la reflexión colectiva.

El espacio como tercer maestro (Castillo-Retamal y Cordeo-Tapia, 2020; Gutierrez, 2005) condiciona los usos y la forma de ser de las personas que los habitan. Asimismo, desde un modelo educativo inclusivo el espacio reivindica su importancia 
como parte del proceso creando así una necesidad incipiente de repensar el uso que se les da a los mismos.

Por tanto, los espacios que habitamos determinan los usos, el desarrollo de capacidades y las identidades. De este modo, el proceso pedagógico y participativo entre los diferentes agentes educativos van construyendo imágenes como excusa para dialogar desde dentro hacia afuera, desde el yo hacia el nosotras.

\section{Referencias}

Castillo-Retamal, F., y Cordero-Tapia, F. (2020). El Tercer Maestro: el espacio natural como catalizador para una educación ambiental efectiva. Revista Saberes Educativos, 4, 48-61. https://doi.org/10.5354/2452-5014.2020.54895

Córdoba, V. Y. (2007). La narrativa visual como metodología del sentido: articulación metodológica e implicaciones terapéutico-educativas. Arteterapia. Papeles de arteterapia y educación artística para la inclusión social, 2, 233-246.

Echavarría, C. V. (2003). La escuela: un escenario de formación y socialización para la construcción de identidad moral. Revista latinoamericana de Ciencias Sociales, Niñez y Juventud, 1(2), 15-43.

Fassina, M., y Torres, M. (2013). Los cuerpos que anidan en los espacios educativos. Dialnet, 29, 49-54.

Gupta, A., y Ferguson, J. (2008). Más allá de la "cultura": espacio, identidad y las políticas de la diferencia. Antípoda. Revista de Antropología y Arqueología, 7, 233-256. https://doi.org/10.7440/antipoda7.2008.10

Gutierrez, K. (2005). Developing a sociocritical literacy in the Third Space: A theoretical Essay. Este artículo fue presentado en AERA Scribner Award Lecture. Montreal, Canada.

Lie, R. (2009). Comprender la hibridación: Hacia un estudio de los espacios de comunicación intercultural. Revista CIDOB d'Afers Internacionals, 88, 43-52.

Mannay, D. (2017). Métodos visuales, narrativos y creativos en investigación cualitativa. Madrid: Narcea.

Olmos, R., Sánchez, D., y Correa, A. (2016). Reflexiones y transformación pedagógica. Reconocimiento a la otredad. Revista Iberoamericana de 
Educación, 71(1), 9-28.

https://doi.org/10.35362/rie71145

Yañez-Urbina, C., Figueroa C., I., Soto, J., y Sciolla H, B. (2018). La voz en la mirada:

Fotovoz como una metodología para explorar los procesos de inclusión-

exclusión desde la perspectiva del estudiantado. Atalanta: Revista de las

Letras Barrocas, 6(2), 1-16.

https://doi.org/10.7764/PEL.55.2.2018.4 